\title{
Breakthrough space obstacle
}

\author{
Bi Qiao1, Song Kongzhi², Zhang Bao Sheng ${ }^{2}$ \\ ${ }^{1}$ Dept. Physics, Wuhan University of Technology, Wuhan 430070, China \\ ${ }^{2}$ Institute of Space Medico-Engineering, Beijing 100192, China
}

\section{Email address:}

biqiao@gmail.com(Bi Qiao)

\section{To cite this article:}

Bi Qiao, Song Kongzhi, Zhang Bao Sheng. Breakthrough Space Obstacle. American Journal of Modern Physics. Vol. 2, No. 4, 2013, pp. 227-233. doi: 10.11648/j.ajmp.20130204.19

\begin{abstract}
In this work, we try to propose a soliton processing mechanism to explain the phenomena of the Breakthrough Space Obstacles (BSO) based on the concrete experimental dates and figures taken from the somatic science. Through analysis and studying, we find that the sort of bio-electromagnetic wave field (BEF) is an origin of BSO. This BEF satisfies a nonlinear quantum Maxwell equation which is proportional to the change of particle density of object. Moreover, BEF has very broad of spectrum range from $100 \mathrm{MHz}$ to $1 \mathrm{GHz}$ and possesses high amplitude of the electromagnetic response, and also has width of frequency as well strong strength. It is this BEF allowing object to transform as a big soliton to have BSO probability by the nonlinear quantum interaction.
\end{abstract}

Keywords: Macro-Tunneling, Soliton, Bio-Electromagnetic Field

\section{Introduction}

The Breakthrough Space Obstacles (BSO) is a term called by the researchers for the macro-object breakthrough a seal up container without broken the wall of the container in the experiments of the somatic science. Although BSO as many phenomena often happened in the experiments of the somatic science [1] in China last 80 s to 90 s, but they were subject to serious critics by standard scientists because the phenomena are hard to be explained by the existence science those days, so that the relevant phenomena existence were suspected [2]. Even so, the two of authors of this work, Song Kongzhi and Zhang Baosheng, had insisted on this sort of experiments by cooperation for 15 years. Many people in the research institute had paid a lot of support in realizing this sort of experiments. The repeatability of the results for BSO have been achieved by using some different sample bottles which officially have special labeled organic glass sheet out by Zhang Baosheng without damage the bottle. We also used the sample bottle and target to unify sealed in a transparent organic glass box which is divided into two layers, the upper bottle placed specimen, the down layer placed out of the target. This sort of BSO had also gotten success [3, 4]. Moreover, the research in the specific function, the breakthrough space disorder phenomena have repeated in many laboratories, and the relevant specific recognition phenomena widely repeated. Here only giving some examples from the different group studying those days: Lin Shuhuang and Liu Huiyi have investigated human specific function phenomena, in their book, the experiment and exposing of human specific functions, they collected 25 experimental papers and 8 theoretical papers with total pages 248 . The twentieth chapter is: the specific tunneling wall phenomenon, the twenty-first chapter is: using micro-light camera takes the observed phenomena of psycho-kinesis and crossing wall. All of that are the repeated experiments about BSO [5]. Wu Banghui group explored the specific functional effects on the silicon crystal structure and gave the assumption of its mechanism [6]. Xiao Guangda group gave a study of the hidden state space and material hidden state, which includes experimental facts of BSO from the carton, wooden and tin containers, and the relevant theoretical assumptions [7]. Zhao Ximing group has studied the functional state of the ECG characteristic specific body for BSO [8]. Zhai Tingxiang group explored changing uncontrollable type specific functionality to controllable type specific features; they found that BSO ability can be cultivated [9]. More marvelously, there were also a series of BSO achieved by Sun Chuling and studied by Shen Jinchuang over 15 years [10], which have arrived at certain high level. Finally even there was a strong united experimental group involved to studying the specific body functions; they also provided a joint experimental report on specific body function authenticity [11]. All of these results provide a conclusion: although BSO seems to be weak for 
the ordinary researchers finding it, but it can be repeatedly by many research groups in different laboratories. As authors of this paper we have been witness that these BSO phenomena were truly existed by the series of evidence of experiments done by Zhang Baosheng and studied by Song Kongzhi for long time [12]. The key problem is that the unknown mechanism of BSO has constructed a serious challenge to modern physics, which led to various critics and suspicions, and enable our many results of BSO to have berried over many years without publication, especially in English version. Even so, the experimental results had received the second reward of Chinese national defense science and technology in 1987, showing some positive support in that period time.

Through long time thinking, in previous work, Bi Qiao and Song Kongzhi have proposed a soliton resonances mechanism to explain the general phenomena of macro-quantum tunneling [13]. In this work, we try to propose a more concrete soliton processing mechanism to explain the phenomena of BSO based on the concrete experimental dates and figures of BSO [12]. This approach may further project a light to reveal this issue through darkness.

\section{Analysis of the Experiment}

Among many evidences of BSO experiment here for simplicity we choose a BSO experiment which was performed by Zhang Baosheng and studied by Song Kongzhi during the first half of 1984. The experimental recording mode used a combination of high-speed camera and video recorder. At that time, we could only buy black and white films which have 27 freeze-frames with 400 meters long because of our funding problem. In order to let the high-speed camera to capture the target image, we used an original packaging of Acanthopanax senticosus bottle. There are 100 tablets of medicine in the bottle, and the tablet diameter is $10 \mathrm{~mm}$.

When the experiment started, after Zhang Baosheng sat on an experimental table and had been quiet, a researcher turned on four 1000 Watt halogen lamps facing to Zhang Baosheng by taking video. The atmosphere began to tense. When people saw Zhang Baosheng threw tablet pills from his hand holding bottle, we immediately started the high-speed camera, and gradually achieved 400 / second speeds. The sound was very large as if train whistle in about 50 square meters laboratory. In order to get a successful image before film running end, we must continue to cheer for encouraging Zhang Baosheng. Many voices intertwined together are simply as if fighting operation! Probably because Zhang Baosheng had already been familiar with us, even in this strong interference situation, he still finally achieved success in the experiment.

Such a long film washing was hard because of that time no one had to wash the black and white film. As it happened, the agricultural film studios copied a batch of old movies, and then through contact, they agreed to help us to wash negative and positive film. Positive film washout showing as movies, we only saw Zhang Baosheng moved much slower than usual, but can not see clearly what place of bottle that the pills went out. Then Song Kongzhi and his research partner Lan Rongliang borrowed an old-fashioned projector and saw the films one by one in a dark room. After looking for a week, they finally saw that a tablet pill was crossing out of the bottle in two pictures. Two of us excited. In a picture, a tablet pill about $2 / 3$ parts has appeared out of the bottle, while the other $1 / 3$ parts remain in the bottle. Its location is very good as front bottom of the bottle. Because it is in the former slightly side, this shows it comes from the bottle. Its position is slightly below, so the image is very clear. The next several pictures from the films clearly show a tablet pill crossing whereabouts process. Another piece of pill is completely on side of the bottle. There are two pictures which illustrate a process of a tablet pill crossing the bottle wall and then gradually falling down. Because it is in the front side of the bottle, so there are two pieces of the whereabouts of the image are on the background of the bottle by producing the background interference. But by looking carefully, this can explain the process clearly.

These films have been completely preserved in the reference room and plan for permanent preservation. The results of this study, fully demonstrates the existence of the specific functions of human body. Because, any false action is in front of a high-speed camera is hard to be hidden during $1 / 400$ seconds. Moreover, it reveals the intermediate state existence of object crossing bottle wall, and reveals that a 10 $\mathrm{mm}$ diameter tablet pill passed an ordinary glass bottle wall in less than $1 / 400$ seconds. This is certainly not seen in general 24 / second film projector. Following a group of Figures 1-8 show a process for a piece of Acanthopanax tablet pill crossing out of a bottom side of the bottle.

Indeed, the first figure shows the original packaging bottle of Acanthopanax senticosus pills; the second one shows that below space of the bottle is empty; the third one illustrates that a half little more pill appears at bottom of the bottle. Special attention should be paid to the interface line between the tablet and the bottom of bottle that is at slightly above bottom of the bottle, which proves that a tablet pill gone out of the bottle is in the front side of the watchers; the fourth one shows the tablet pill has been out of the bottle; the fifth one demonstrates the tablet pill is continuing to fall, but has not yet reaches the desktop. The following Figures 6-8 are the amplification of Figures 2-4, respectively. As we can see, in the pictures, the hand posture and relative position of the Zhang Baosheng are very stable and only a little change. That is, Zhang Baosheng's right hand holding the bottle, from the second photo to the fifth photo, after 3/400 seconds, slightly elevated. In the second photo, the bottle is covering his sleeve, and at the fifth photo, the bottle below exposes a finger width of his sleeve shadow. The shadow of the bottle also moves with lamplighter moves right. This is all possible movement of him during 3/400 seconds, which fully demonstrates objectivity of fact that a tablet pill moved out the bottle through the wall of bottle during this period of 
time.

After the object come breakthrough space obstacle, whether the container physical changes is always an important topic to our attention. Due to the experimental conditions at that time, the changes of contents of object was mainly rely to observing. As the content of sodium chloride, silver, lithium fluoride and silicon, after their BOS took place from a sealed bottle, the molecular structure of the sodium chloride, silver, and lithium fluoride were no change, but the crystal structure of monocrystalline silicon has changed by measurement of X- ray diffraction method, so the orientation of crystallites structure becomes to the minicrystal and the original peak structure becomes to the flat. But this change disappeared after past a week. The change was a kind of soft change.

From above description of the experiment, we can give following conclusions: (1) The BSO process is physics, not psychology. (2) BSO is directly to tunnel the wall of container in our four dimensional space-time, not in else dimensions. (3) BSO moves out of the wall of container at certain velocity, a pill with $1 \mathrm{~cm}$ diameter passes through the transparent glass container during 0.005 seconds, namely the speed is about $200 \mathrm{~cm} / \mathrm{sec}$. For a paper bag with length of 5 $\mathrm{cm}$ passes through the transparent glass bottle wall costs 0.12 seconds, the speed is about $41.7 \mathrm{~cm} / \mathrm{sec}$. So from the camera and high-speed photography information, the BSO speed should be between $40-200 \mathrm{~cm} / \mathrm{s}$. (4) The object is influenced by the sort of field from (the hands of) Zhang Baosheng to have ability of BSO; (5) After BSO, the container is still seal up without broken.

All of above analysis points to a physical concept: quantum tunneling. However, as we know the object is a macro-object which is not quantum and the classical object can not tunnel through an infinite potential well based on the fundamental physics, there is a big contradiction between phenomena and physics, which enables one hard to imagine.

However, there still exists possibility. The key concept is macroscopic quantum effect influenced by the radiation electromagnetic wave field from the subject. In terms of the previous works of Bi Qiao, we have proposed that these sorts of phenomena may happen based on the so called macro-quantum effect, such as soliton realizing processing $[14,15]$. Hence in following sections we try to propose a mechanism to explain BSO based on the above experiment.

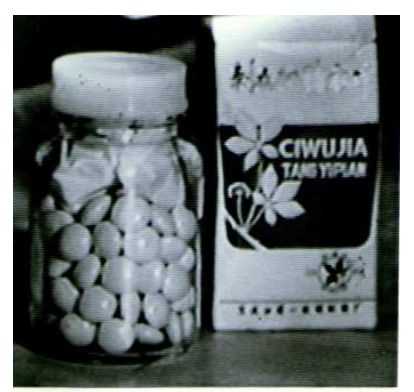

Figure 1. Acanthopanax senticosus raw package bottle

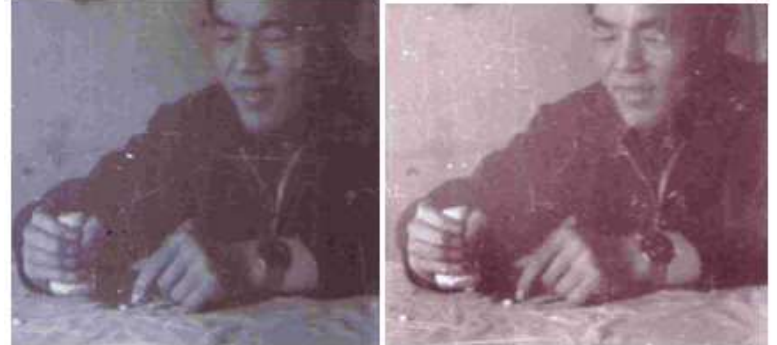

Fig.2 The bottle below space is empty; Fig.3 A half piece of one pill has appeared to the bottle below. Special attention should be paid to the interface line of pill and bottle that is slightly above bottle bottom. That tablet pill is crossing out of front side of bottle facing to reader.

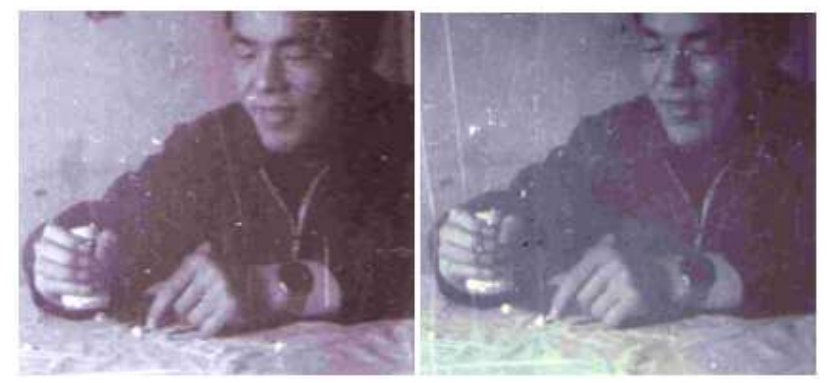

Fig. 4 A tablet pill has been out of the bottle; Fig.5 A tablet pill continues to fall, but has not yet reached the desktop.
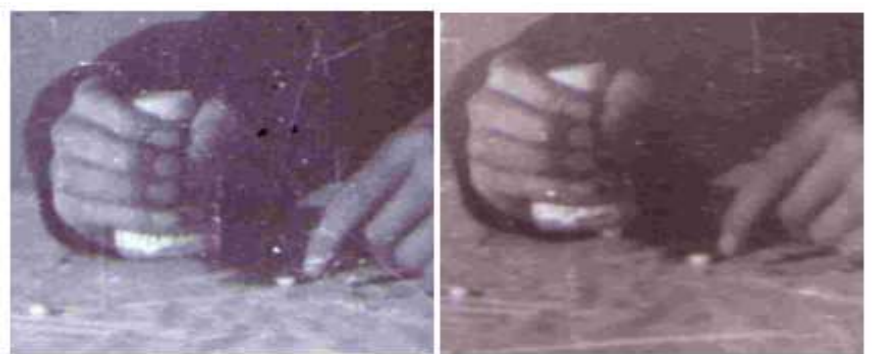

Fig.6 is Fig. 2 amplified; Fig. 7 is the Fig. 3 magnified.

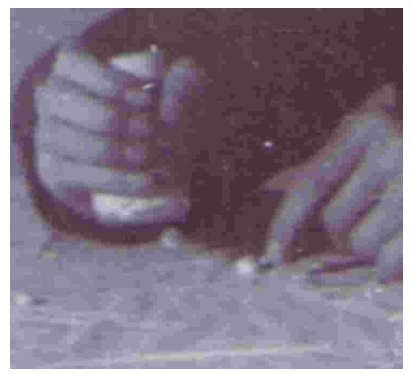

Fig. 8 is Fig. 4 magnified.

\section{Mechanism Proposed}

The proposed main ideas are: (1) the object in the bottle is subject to a sort of influence of the bio-wave field produced by the subject (Zhang Baosheng); (2) this bio-wave field interacts with the object, by which the object becomes to a soliton; (3) this soliton can tunnel out to bottle through the wall.

Indeed, the object can be described by a Schrödinger equation of many body systems, under the total wave 


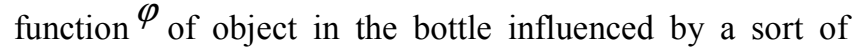
nonlinear interaction from an external bio-field $F$ through a coupling $\chi$, the equation is written by

$$
i \frac{\partial}{\partial t} \varphi=\frac{1}{2 m} \nabla^{2} \varphi+V(x, t) \varphi+\chi \varphi F
$$

where the bio-field is provided by the subject described as

$$
\frac{\partial^{2} F}{\partial t^{2}}-v_{0} \frac{\partial^{2} F}{\partial x^{2}}=-\chi \frac{\partial^{2}}{\partial x^{2}}|\varphi|^{2}
$$

This shows that the total wave function $\varphi_{\text {is distorted }}$ with the influence of the $F$ through coupling $\chi$, while the change of the particle density $\partial^{2}|\varphi|^{2} / \partial x^{2}$ influence the status of $F$ as

$$
F=-\frac{\chi^{2}}{v^{2}-v_{0}^{2}}|\varphi|^{2}
$$

which gives a nonlinear Schrödinger equation as

$$
i \frac{\partial}{\partial t} \varphi=\frac{1}{2 m} \nabla^{2} \varphi-V(x, t) \varphi-g|\varphi|^{2} \varphi
$$

with

$$
g=-\frac{\chi^{2}}{v^{2}-v_{0}^{2}}
$$

where $v\left(v_{0}\right)$ is an (initial) velocity of field propagation. Then a soliton type of solution exists. For example, if $V(x, t)=0$, and $g$ is a constant, then a solution can be given as well known [16]

$$
\begin{aligned}
& \phi(x, t) \\
& =B_{0} \operatorname{sech}\left\{B_{0} \sqrt{m g}\left[\left(x-x_{0}\right)-v_{0}{ }^{\prime} t\right]\right\} \exp \left\{i m v^{\prime}\left[\left(x-x_{0}\right)-v^{\prime} t\right]\right\}
\end{aligned}
$$

where defining

$$
\begin{aligned}
& x^{\prime}=x \sqrt{2 m} \\
& B_{0}=\sqrt{\frac{v^{\prime}-2 v_{0}^{\prime} v^{\prime}}{g}}
\end{aligned}
$$

here $v^{\prime}=d x^{\prime} / d t$ is a velocity of soliton, and $v_{0}{ }^{\prime}$ is an initial velocity of the soliton. This means under the nonlinear interaction from the bio-field, the object becomes a big soliton with highly coherence, which can be described by one wave function, soliton $\varphi(x, t)$, while the $F$ influence the shape, velocity, and amplitude of the soliton through ${ }^{g}$ as a function of $\chi$ and $v$. The probability of macro-quantum tunneling of the solitons have discussed in ref.[13]. In the above experimental situation the object soliton is confined in the potential well (the bottle), which is described by a type of the Grosse-Pitaevskii equation as Eq.(4). Then there exist BSO happening as a distribution of the wave functions between inside and outside of the bottle, which are supposed as

$$
\phi(\mathbf{r}, t)=a(t) \phi_{1}(\mathbf{r})+b(t) \phi_{2}(\mathbf{r})
$$

where $\phi_{1}(\mathbf{r})$ or $\phi_{2}(\mathbf{r})$ is the wave function of the soliton in or out of the bottle, respectively, and the corresponding $a(t)$ or $b(t)$ is a probability amplitude in or out of the bottle. Then, by using Eq.(4) again, one gets the solution as

$$
\begin{aligned}
& |a|^{2}=\left|f_{a}\right|^{2}\left|a^{\prime}\right|^{2} \\
& =\frac{1}{2 c} \frac{\sqrt{\gamma^{2}+v^{2}} \sin ^{2}\left(\frac{t}{2} \sqrt{\gamma^{2}+v^{2}}\right)}{\left(\frac{t}{2} \sqrt{\gamma^{2}+v^{2}}\right)-\sin \left(t \sqrt{\gamma^{2}+v^{2}}\right)}
\end{aligned}
$$

$$
\begin{aligned}
& |b|^{2}=\left|f_{b}\right|^{2}\left|b^{\prime}\right|^{2} \\
& =\frac{1}{2 c} \frac{1-\frac{v^{2}}{\gamma^{2}+v^{2}} \sin ^{2}\left(\frac{t}{2} \sqrt{\gamma^{2}+v^{2}}\right)}{t-\frac{v^{2}}{\left(\gamma^{2}+v^{2}\right)^{\frac{3}{2}}}\left[\frac{t}{2} \sqrt{\gamma^{2}+v^{2}}-\sin \left(t \sqrt{\gamma^{2}+v^{2}}\right)\right]}
\end{aligned}
$$

which reveals that the object tunneling behavior is as a complicated inharmonic oscillation with many frequencies. One of amplitudes is

$$
\frac{\sqrt{\gamma^{2}+v^{2}}}{2 c}
$$

showing $c, \gamma$, and $v$ increase with the amplitude decrease. Where notice here $\gamma=\gamma_{1}-\gamma_{2}$ and $v$ may not relate to $V_{\text {ext }}$,

$$
\begin{aligned}
& \gamma_{j}=\int \frac{1}{2 m}\left|\nabla \varphi_{j}(r)\right|^{2} d r, j=1,2 \\
& v=2 \int \frac{1}{2 m} \nabla \varphi_{1}(r) \nabla \varphi_{2}(r) d r
\end{aligned}
$$

here

$$
c=g \int|\varphi|^{4} d r
$$

reflects the nonlinear coupling. When adjusting

$$
\sin ^{2}\left(\frac{t}{2} \sqrt{\gamma^{2}+v^{2}}\right)=0
$$

and

$$
\frac{t}{2} \sqrt{\gamma^{2}+v^{2}}=n \frac{\pi}{2}
$$


the BSO can take place completely. This supports that the object can realize $\mathrm{BSO}$ as a macro-quantum tunneling effect by giving the momentum through shaking the bottle by Zhang Baosheng as shown in the experiment. Furthermore, considering $\gamma,{ }^{\gamma}$ is continuous, tunneling time ${ }^{t}$ determined by Eq.(17) is like quantum, which means the intermediate states of tunneling are difficult to keep because they are also quantum. This seems to be supported by the fact that Zhang Baosheng was hard to hold the intermediate status during BSO happening [3]. In a studying from above formalism, we find that the change of $F$ given by the Eq.(3) demonstrates certain resonance between the wave field projected by Zhang Baosheng and the particle density of object, which enables a nonlinear Schrödinger equation to be achieved again as Eq.(4). So based on this equation, we see that the bio-field $F$ is important to realize the type of soliton for the object in the sense: (1) $F$ is a sort of physical wave field, (2) this wave field must be proportional to the particle density of the object, (3) this wave field must be strong enough. The essential conditions are (2) and (3) which normally are hard or even impossibly to realize for people; fortunately, they are possibly performed by some one who possesses the specific functions such as Zhang Baosheng.

As investigation of this sort of bio-wave field, in that period time Song Kongzhi considered an experiment to measure the spectrum of external electromagnetic wave of Zhang Baosheng, shown in Fig.9, where Zhang Baosheng even used his gentle touch hands "screwed" bending stainless steel forks and spoons, (whose function is the ordinary people can not be completed), but interestingly, when Zhang Baosheng completed this function by concentrating his attention to the forks and spoons, there was not record of the electromagnetic wave showing. Only, when Zhang Baosheng concentrated on antenna or shielding line to send his "wave", the radiation of electromagnetic wave was recorded. This may indicate that his radiation of electromagnetic wave possesses strongly concentrated direction and target. The Fig.9 illustrates that the change domain of the electromagnetic wave of Zhang Baosheng. The first chart of left shows the experiment of Zhang Baosheng on the morning of August 20, 1997; the above curve as the background with five averages; below curve represents for the Zhang Baosheng power curve, with the distance spiral antenna $50-100 \mathrm{~cm}$; the starting point of horizontal ordinate is $1 \mathrm{MHz}$, the ending point is $1 \mathrm{GHz}$ as the logarithmic curve, i.e. $1,2,3 \ldots 10 \mathrm{MHz}, 10,20,30 \ldots$ $100 \mathrm{MHz}, 100,200,300 \ldots 1 \mathrm{GHz}$; in vertical axis, the top line is $-70 \mathrm{dBm}$, each 1 down to $2 \mathrm{~dB}$; below expression is as marker, in the diagram is $169.27 \mathrm{MHz}$. The upper right corner marked the point values of frequency and intensity, strength as $-84.244 \mathrm{dBm}$; the lower left corner is respectively RBW (Resolution bandwidth), general scanning frequency highest value 1/1000; VBW (Video band width) is for video bandwidth, often scanning frequency of $1 / 1000$, considered as the scanning accuracy; SWP (sweep time) is for scanning time. These parameters can be set according to the purpose and conditions, where in the charts: RBW: $1 \mathrm{MHz}, \mathrm{VBW}: 1$ $\mathrm{KHz}$, SWP: 5 seconds, and the measuring range is $10 \mathrm{MHz}-1 \mathrm{GHz}$. There are two measured curves, a curve above stands for background, and below one expresses actual strength curve of electromagnetic wave for the subject after subtracting background intensity. The figures on the left 1, 2 and on the right 1, 3 represent Zhang Baosheng in three experimental results on the morning of August 20, 1997. The two figures on the left describe Zhang Baosheng projected power using hand with distance from antenna $50-100 \mathrm{~cm}$. A picture on the right is the result showing Zhang Baosheng from antenna $50-100 \mathrm{~cm}$ with hands and mouth both to send powers. Right on the second picture represents one who has no function but only simulated Zhang Baosheng power action. As comparison we can see in this situation there is only a little induction wave with slightly strengthen in a peak of the background map. Moreover, there are four small figures in the diagram below to show the measurement results for Zhang Baosheng on the morning of August 22, 1997. Measuring conditions were the same as above, only the highest lines on the map is $-40 \mathrm{dBm}$, each small lattice $5 \mathrm{dBm}$. As we see the measuring strength has increased $30 \mathrm{dBm}$. Zhang Baosheng used both hands and mouth to send power with wide frequency range and widespread radiation waves.

Hence, from the curves in Fig.9 one can find that the spectrum range of the electromagnetic radiation response is very broad, from $100 \mathrm{MHz}$ to $1 \mathrm{GHz}$; there are high amplitudes of the electromagnetic response. These frequency width and strong strength are impossibly measured by several of simulations. The signal of Zhang Baosheng can be higher $47 \mathrm{dBm}$ than environment with the absolute value reaching to $37 \mathrm{dBm}$. This was also higher $5 \mathrm{dBm}$ than the TV station signal strength measured by opening the door in the laboratory. Furthermore, the radiation wave shapes of Zhang Baosheng measured were not the same as TV station. Some are uniformed modals, showing a single steep peak and peak asymmetry. Some are slow peaks, the peaks are not high, and the frequency ranges are wide. Some are comprehensive with high amplitudes, wide frequency band and no fixed location of perplexing waveform.

In particular, before the experiment, the various of analog signals, for instances 3 volt battery produces positive and negative electrode tip discharge, emission of mobile phone and walkie-talkie transmitter, television transmitter, environmental chemical fiber fabric friction etc., had been measured by Song Kongzhi and the researchers. The electromagnetic waves produced in all of these cases were different from that of Zhang Baosheng. The transmitted waveform of mobile phone, walkie-talkies and TV station are generally very order and standard uniformed modal waves, while the amplitude of friction discharge of dry chemical fiber or positive and negative point discharge of battery is very small and very clutter. Hence, the experiments show that, in the power state of Zhang Biaosheng, he can produce a sort of complicated 
electromagnetic wave radiation.

Considering the support facts from the above experiment, we believe that the wave field $F$ is a sort of bio-electromagnetic field (BEF) in this BSO experiment, which satisfies Eq.(2), that means $F=A$ is a kind of vector potential, and Eq.(2) is a kind of nonlinear quantum Maxwell equation [17]. This sort of BEF has very broad of spectrum range as $100 \mathrm{MHz}$ to $1 \mathrm{GHz}$ and possesses high amplitude of the electromagnetic response. It has width of frequency with strong strength. All of these keep the electromagnetic wave of BEF to easily pursue the change (or resonance) of density of particles, and influence the shape, velocity, amplitude of the soliton of the object through $g$ to realize the BSO.

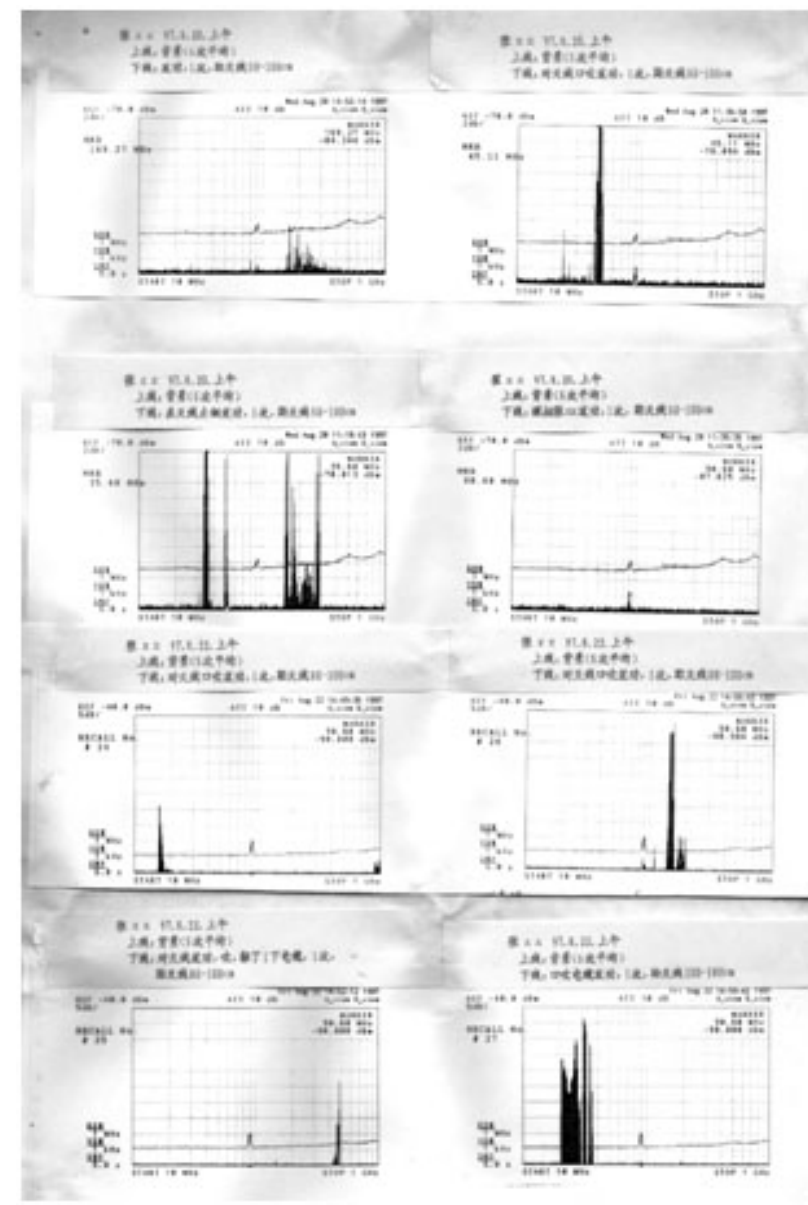

Fig. 9 This was Zhang Baosheng in vitro electromagnetic wave recorded in April 20-22, 1997 in completing the experiment. From the change of the electromagnetic wave curve we can see the electromagnetic radiation response spectrum range is very broad, from $100 \mathrm{MHz}$ to $1 \mathrm{GHz}$ there are electromagnetic response of high amplitudes. The frequency width and strong strength are not possibly measured by other various simulations. Zhang Baosheng electromagnetic wave can be higher than $47 \mathrm{dBm}$ environment, its absolute value can reach $37 \mathrm{dBm}$ and is also higher $5 \mathrm{dBm}$ than TV signal strength measured by opening door in the laboratory. Moreover, these radiation wave shapes measured from Zhang Baosheng are not exactly the same as the shapes of other sort of electromagnetic wave signals because some are unimodal, showing a single steep peak with peak asymmetry; some have slow peak and the peak are not high, and the frequency range are wide; some are a comprehensive, with high amplitude and wide frequency band without fixed location of complex waveforms.

\section{Conclusions}

In conclusions, the concrete BSO experiment is described and analyzed. The background of experiments for BSO is introduced. Based on the experimental dates and photos, a mechanism of BSO is proposed. The sort of BEF produced by Zhang Baosheng is an origin of BSO. This BEF satisfies a nonlinear quantum Maxwell equation which is proportional to (or is certain resonance with) the change of particle density of object. Moreover BEF has very broad of spectrum range $(100 \mathrm{MHz}$ to $1 \mathrm{GHz})$, possesses high amplitude of the electromagnetic response and owns width of frequency as well holds strong intensity.

\section{References}

[1] Song Kongzhi, The Existence and Significance of Parapsychological Function, J. International Society of Life Information Science (ISLIS) 17(1) 1999, pp. 198-214.

[2] Song Kongzhi, Lan Rongliang, Li Xianggao, and Zhou Liangzhong, The Research of the break through spacial obstacle function, Create somatic science, 705-717, Sichuan Educate Publisher, Beijing, 1989.

[3] Song Kongzhi, et al., Observation of large container the breakthrough space obstacle, J. Chinese Somatic Science 1(4) 1991, pp. 163.

[4] Song Kongzhi, and Zhang Baosheng, Several specific functions and comparison, J. Chinese Somatic Science 3(3) 1998, pp 99.

[5] Lin Shuhuang, and Liu Huiyi: Test and explore human phenomenon, China Radio International Press, China Beijing, published in November 1997.

[6] Wu Banghui: a preliminary discussion of specific functional effects on the silicon crystal structure and its mechanism, $J$. Chinese Somatic Science 1(4) 1991, pp.160.

[7] Xiao Guangda, et al., A study of the hidden state space and material hidden state, J. Chinese Somatic Science 1(4) 1991, pp. 178.

[8] Zhao Ximing, et al. A study of the functional state of the ECG characteristic specific body, J. Chinese Somatic Science 3(3) 1998, pp. 114.

[9] Zhai Tingxiang, et al. Change the uncontrollable type specific functionality to a controllable type specific features, $J$. Chinese Somatic Science 4(2) 1999, pp. 73.

[10] Feng Bai, Chulin Sun, Zhiyi Liu, and Jinchuan Shen, Yin Zhu, Rongchao Ge, Caili Bei, Jindong Zhang, Xiaohong Shi, Yicheng Liu, Xuecheng Liu, Seeds Induced to Germinate Rapidly by Mentally Projected "Qi Energy" are Apparently Genetically Altered, American Journal of Chinese Medicine 28(1) 2000, pp. 3-8.

[11] Joint test group for studying the specific body function, The joint test report on specific body function of authenticity, $J$. Chinese Somatic Science 9(4) 1999, pp. 160.

[12] Song Kongzhi, Zhang Baosheng specific function of the enlightenment, Unpublished (in Chinese), 2009. 
[13] Bi Qiao, and Song Kongzhi, Macroscopic Quantum Tunneling, Journal of Modern Physics 4 2013, pp. 49-55.

[14] Bi Qiao, Bio-Solitons Return Life, International Review of Physics 3(5) 2009, pp. 278-288.

[15] Bi Qiao, Song Kongzhi and H. E. Ruda, Characteristics of coherence and information for the Davydov soliton field, $J$
Modern Phys. 3 2012, pp. 1907-1913.

[16] Pang Xiao-Feng, and Feng Yuan-Ping, Quantum Mechanics In Nonlinear Systems, World Scientific Publishing Co. Pte. Ltd. Singapore, 2005.

[17] Mark Srednicki, Quantum Field Theory, Cambridge University Press, 2007. 